PAPER • OPEN ACCESS

Effects of the initial axial strain state on the response to transverse stress of high-performance RRP $\mathrm{Nb}_{3} \mathrm{Sn}$ wires

To cite this article: J Ferradas Troitino et al 2021 Supercond. Sci. Technol. $\mathbf{3 4} 035008$

View the article online for updates and enhancements.

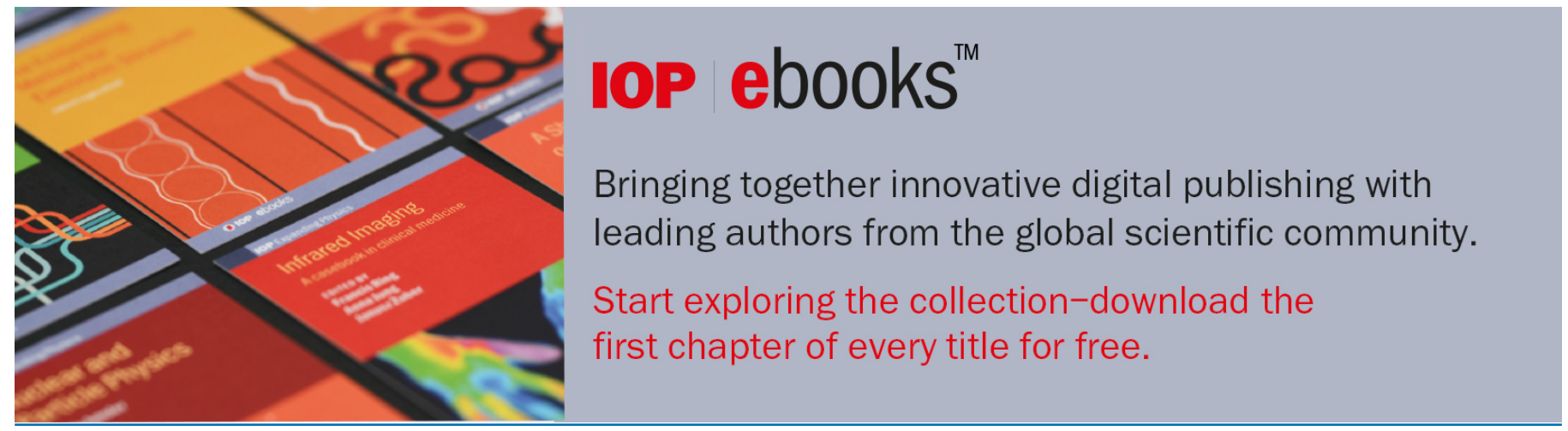

This content was downloaded from IP address 188.185 .86 .226 on $08 / 02 / 2021$ at $10: 34$ 


\title{
Effects of the initial axial strain state on the response to transverse stress of high-performance RRP $\mathrm{Nb}_{3} \mathrm{Sn}$ wires
}

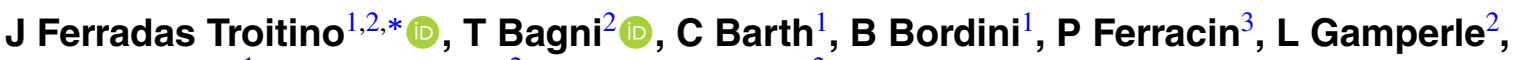 \\ D Tommasini ${ }^{1}$, D Zurmuehle ${ }^{2}$ and C Senatore ${ }^{2}$ (1) \\ ${ }^{1}$ CERN, The European Center for Nuclear Research, Geneva 23, 1211, Switzerland \\ ${ }^{2}$ University of Geneva, Geneva, Switzerland \\ ${ }^{3}$ Lawrence Berkeley National Laboratory, Berkeley, CA 94720, United States of America \\ E-mail: jose.ferradas.troitino@ cern.ch
}

Received 1 September 2020, revised 1 December 2020

Accepted for publication 14 December 2020

Published 29 January 2021

\begin{abstract}
High-performance $\mathrm{Nb}_{3} \mathrm{Sn}$ superconducting wires have become one of the key technologies for the development of next generation accelerator magnets. While their large critical current densities enable the design of compact accelerator-quality magnets for their operation above $10 \mathrm{~T}$, the noticeable reduction of the conductor performance due to mechanical strain appears as a new essential characteristic in magnet design.

In this work, we extensively investigate the effect of transverse loads, up to $250 \mathrm{MPa}$, in state-of-the-art $\mathrm{Nb}_{3} \mathrm{Sn}$ Restacked-Rod-Process round superconducting wires. The tests are performed using a compressive Walters spring device, where the force is applied to the resin-impregnated wire, and the critical current is measured under magnetic fields ranging from 16 to $19 \mathrm{~T}$. As a complement, critical current measurements under axial strain are also performed using a standard Walters spring. Interestingly, the study shows that the wire's electro-mechanical response under transverse stress depends on the initial axial strain condition. Nonetheless, when the main direction load becomes predominant, all tested wires converge to a common behavior. This observation allowed us to combine the results from critical current measurements under the loads exerted in both directions (axial and transverse), shedding some new light on the mechanisms behind critical current degradation.
\end{abstract}

Keywords: $\mathrm{Nb}_{3} \mathrm{Sn}, \mathrm{RRP}$, strain sensitivity, critical current degradation, transverse strain, axial strain

(Some figures may appear in colour only in the online journal)

\section{Introduction}

The search for high magnetic fields in superconducting accelerator magnets has contributed over the last decades to several

Author to whom any correspondence should be addressed.

Original Content from this work may be used under the terms of the Creative Commons Attribution 4.0 licence. Any further distribution of this work must maintain attribution to the author(s) and the title of the work, journal citation and DOI. important technological breakthroughs. One of the keys for this evolution has been the development of practical superconductors able to operate beyond the limits of the wellknown $\mathrm{Nb}$-Ti technology. Due to their larger upper critical field, $\mathrm{Nb}_{3} \mathrm{Sn}$ (Niobium-Tin) conductors have become in the present days the preferred solution for the winding of the new generation superconducting coils.

High performance $\mathrm{Nb}_{3} \mathrm{Sn}$ wires are primarily defined by large critical current densities, an essential parameter that permits the design of compact-size magnet units. Thanks to it, and accompanied by comprehensive programs for conductor development, the use of this new technology has unlocked the 
previous magnetic field frontiers and has allowed the design of accelerator magnets for their operation above $10 \mathrm{~T}[1,2]$.

The first milestone for $\mathrm{Nb}_{3} \mathrm{Sn}$ applications in High Energy Physics (HEP) research programs will be achieved with the installation of the Niobium-Tin dipole and quadrupole magnets built for the High-Luminosity upgrade of the LHC (HL-LHC) at CERN [3]. These will be the first $\mathrm{Nb}_{3} \mathrm{Sn}$ magnets ever operating in a particle accelerator and will pave the way towards potential future hadron colliders seeking for larger collision energies, as it is the case for instance, of the Future Circular Collider (FCC) [4].

The HL-LHC conductors constitute the present state-ofthe-art for $\mathrm{Nb}_{3} \mathrm{Sn}$ accelerator technology. They have their origin in the research programs started in the mid-1990s, and extended over the following decades [5]. During the first part of that period, the critical current density within the noncopper wire area $\left(J_{c}\right)$ was substantially pushed from nearly $2000 \mathrm{~A} \mathrm{~mm}^{-2}$ to above $3000 \mathrm{~A} \mathrm{~mm}^{-2}$ at $12 \mathrm{~T}$ and $4.2 \mathrm{~K}$. In the course of the next years after this important achievement, further efforts consolidated the conductor performance and made possible the attainment of long production lengths. As a result, nowadays the wires have demonstrated, with large margin, the capability of surpassing the target of $J_{c}>1000 \mathrm{~A} \mathrm{~mm}^{-2}$ at $16 \mathrm{~T}$ and $4.2 \mathrm{~K}$ while maintaining the necessary properties for stable operation and magnet protection [6-9].

An equally important aspect of $\mathrm{Nb}_{3} \mathrm{Sn}$ superconductors is, however, their reduced mechanical strain tolerance. The critical current density of $\mathrm{Nb}_{3} \mathrm{Sn}$ wires strongly depends on the mechanical strain applied to the superconducting phase [10-27], this last being, in addition, a brittle intermetallic compound with a noteworthy propensity to fracture. For the most relevant cases in magnet design, the action of mechanical loads translates consequently into a reduction of the conductor performance, i.e. a reduction of the wire's $J_{c}$.

The mentioned decrease of the available critical current density happens in two well-distinguished regimes. On one side, there is a reversible component of the reduction, which is fully recovered when the external load is removed, and is associated to the decrease of the upper critical field $\left(\mathrm{B}_{c 2}\right)$ under mechanical strain [19]. On the other side, there exists an irreversible and thus permanent component, which occurs when the loads exceed a critical threshold, and that can be ascribed to plastic deformation in the wire's copper matrix and to superconducting filament breakage [28]. In a scenario where the design of present and next generation accelerator magnets entails peak stresses in the coils that are in the level of $150-200 \mathrm{MPa}$ [29], the degradation of the conductor performance due to mechanical strain appears to be a parameter of the highest importance for new $\mathrm{Nb}_{3} \mathrm{Sn}$ magnets [30].

Establishing the mechanical limits at which $\mathrm{Nb}_{3} \mathrm{Sn}$ superconductors can operate safely, and understanding the mechanisms behind critical current degradation is hence crucial, and requires the development of dedicated experiments able to reproduce the conditions experienced by the conductor inside superconducting magnets. Performing such a task is nonetheless a very involving activity due to the complexity of the system. As far as $\mathrm{Nb}_{3} \mathrm{Sn}$ accelerator magnet's coils are concerned, they are in a large extent wound using insulated Rutherford cables composed of many single wires transposed together. These cables are ultimately resin-impregnated, conforming a solid winding pack intended for an homogeneous distribution of the mechanical loads inside the coils. In what regards the loads, the cables and by extent the wires, are subjected to different types of them, appearing both during magnet construction and operation. In general, restricting ourselves to the operation regime, the most severe loads are the ones acting in the cables' transverse direction (orthogonal to the cables' broad face) and usually located in the coils' mid-planes of new high field designs. The study of their effects in conductor performance is, by implication, of special interest for the magnet community.

The first designs for experimental setups aimed at testing the conductor response to mechanical loads date from the early 1980s by Ekin, who performed novel critical current measurements under tensile axial strain for short wire sections [11]. Since then, different strategies have been developed for evaluating the impact of mechanics in the critical current $\left(I_{c}\right)$ of $\mathrm{Nb}_{3} \mathrm{Sn}$ superconductors [31]. Related to accelerator magnet applications, they range from critical current measurements in full cables (or sub-scale cables) down to single wires, on which the conductor is subjected to the action of a certain type of load [32-50].

Employing the latter type of these experiments, we have launched at University of Geneva a complete test campaign for the investigation of the response of $\mathrm{Nb}_{3} \mathrm{Sn}$ wires to transverse loads [30]. The scope of the study is to analyze the strain dependence and the irreversible reduction of the critical current of single impregnated wires, when tested in conditions that reproduce their state inside accelerator coil windings. To do so, we make use of a test setup based on a modified Walters spring, as will be explained in detail in section 2. Interestingly, from the first results obtained, we have found that the electro-mechanical response of $\mathrm{Nb}_{3} \mathrm{Sn}$ impregnated wires to transverse strain is determined by a combination of different factors, which are intrinsic and extrinsic to the wires. They include: (i) the way mechanical loads are applied to the wire and the wire's geometry (round, or 15\% rolled reproducing the geometry of the wires present in superconducting cables), (ii) the wire technology and its filament layout, and (iii) the stiffness of the resin system used for impregnation [28, 51].

In this manuscript, we continue the work initiated for Powder-In-Tube (PIT) conductors in [30], and we extend it to the electro-mechanical characterization of state-of-the-art $\mathrm{Nb}_{3} \mathrm{Sn}$ Restacked-Rod-Process (RRP) wires. We report on the first results from tests performed in a set of round samples of the same wire. For them, critical current measurements under loads applied in transverse direction were performed in presence of magnetic fields ranging from 16T to 19T. We have identified an important role of the axial strain component in the response of the wires to transverse loads. In view of it, the transverse measurements have been combined with critical current measurements under axial strain, obtaining a complete description of the phenomenology. Our work covers as well two other fundamental aspects: (1) the identification of the total reduction of critical current experienced under transverse stresses up to $250 \mathrm{MPa}$ and (2) the determination of the stress 


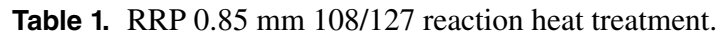

\begin{tabular}{ll}
\hline Temperature plateau & Ramp rate \\
\hline $210^{\circ} \mathrm{C} / 48 \mathrm{~h}$ & $25^{\circ} \mathrm{C} / \mathrm{h}$ \\
$400^{\circ} \mathrm{C} / 48 \mathrm{~h}$ & $50^{\circ} \mathrm{C} / \mathrm{h}$ \\
$665^{\circ} \mathrm{C} / 50 \mathrm{~h}$ & $50^{\circ} \mathrm{C} / \mathrm{h}$ \\
Cool down & $100^{\circ} \mathrm{C} / \mathrm{h}$ \\
\hline
\end{tabular}

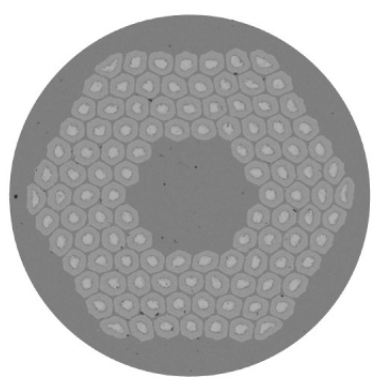

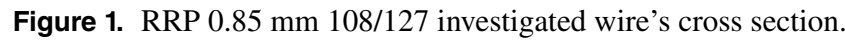

level at which permanent damage occurs in the wires. The results add new information to the main conclusions already published for PIT conductors, and shed some new light on the mechanisms behind the degradation mechanisms in the wires produced by RRP technology.

\section{Experimental campaign description}

A set of identical RRP $\mathrm{Nb}_{3} \mathrm{Sn}$ samples, extracted from one of the production billets to be used for the new HL-LHC $\mathrm{Nb}_{3} \mathrm{Sn}$ interaction region quadrupole magnets (known as MQXF) [52], were investigated during this experimental campaign. With a diameter of $0.85 \mathrm{~mm}$, the wires are composed of 108 sub-elements embedded in a high-purity $\mathrm{Cu}$ matrix following the 108/127 hexagonal restack [53]. The nominal resulting copper to superconductor ratio $(\mathrm{Cu} / \mathrm{SC})$ is specified to be 1.2. Reproducing what is done for MQXF superconducting coils, the samples have been reacted following the reaction heat treatment indicated in table 1 . A picture of the wire cross-section before reaction is shown in figure 1.

Concerning the measurements, three types of tests at cryogenic temperature $(4.2 \mathrm{~K})$ have been performed as part of the characterization campaign: (1) critical current measurements under compressive transverse loads, (2) critical current measurements under axial loads, and (3) stressstrain measurements for the study of the wires' mechanical properties.

\subsection{Compressive WASP (C-WASP) measurements}

The transverse compressive tests were carried out using a measurement device with a geometry similar to a Walters spring (referred as C-WASP) [28, 54, 55], where the force is applied to the resin-impregnated wire and the critical current is measured under magnetic fields ranging from 16T to 19T. As it can be seen in figure 2 , which shows a three dimensional model of the test setup, the sample holder is composed of two parts: a movable lower part, able to translate axially, and a top part, which is fixed to the measuring probe. The transverse force is applied by means of a pulling rod that acts on the lower part of the sample holder, displacing it towards the top fixed anvil and applying the desired compression. The anvil extends over a length of $126 \mathrm{~mm}$, defining the U-shaped cavity where the impregnated wire sits. The rest of the cavity's dimensions can be adjusted for different wires as function of their diameter. For the presented case, the cavity remains $1.15 \mathrm{~mm}$ in width and $2 \mathrm{~mm}$ in height. Regarding the sample impregnation, it has been performed using a de-gassed mixture of epoxy resin (type $\mathrm{L}+$ hardener $\mathrm{L}$ provided by $\mathrm{R} \& \mathrm{G}$ Faserverbundenwerkstoffe) and filler (thixotropic agent provided by SCS-Füllstoffe) in a 100:40:2 weight ratio. The choice for this impregnation system relies on its favorable characteristics for manipulation and on its similar measured elastic modulus, when compared to the one of CDT-101 K resin used for the impregnation of HL-LHC $\mathrm{Nb}_{3} \mathrm{Sn}$ coils $[56,57]$. To ensure a proper current transfer, the wire is soldered over $250 \mathrm{~mm}$ to the sample holder extremities. Finally, the superconducting to normal transition is measured using voltage taps, which are placed at the entrance and exit of the groove. They are separated by the distance of a complete wire turn, $126 \mathrm{~mm}$. This large gauge length allows us to use the stringent criterion of $0.1 \mu \mathrm{V} \mathrm{cm}^{-1}$ for critical current determination.

\subsection{Axial WASP (WASP) measurements}

Critical current measurements under axial strain were performed using a Walters spring (WASP) device. In this case, a one-piece sample holder with the geometry of a thick spring hosts the wire as shown in figure 3 . The desired axial strain is applied to the sample as a result of the spring deformation, induced by a controlled rotation of one of its extremities. The rotation angle determines the strain level, while its direction specifies the compressive or tensile behavior. A more detailed explanation on how the device works can be found in [58]. For the tests reported in this manuscript, the axial WASP measurements were done at $15 T<B<19 T$ with two different objectives: (1) to investigate the wire's critical current as function of the applied axial strain and (2) to determine the value of critical current at the the wires' zero applied axial strain state $\left(I_{c 0} \varnothing\right.$ strain $)$. Precisely, the zero applied strain state corresponds to a test condition that ensures that no external loads are exerted to the wire, and therefore, the superconducting phase is just subjected to the mechanical strain arising from the thermal contraction mismatch between the different materials of the composite wire. While the first type of measurement follows a standard procedure, the second one presents some particularities that are carefully explained in [59].

In all cases, and like in the transverse experiment, the criterion for critical current determination is set to $0.1 \mu \mathrm{V} \mathrm{cm}^{-1}$, where four voltage tap pairs are placed along the spring path. Each of them separated by a distance equal to the length of a full turn $(126 \mathrm{~mm})$. 
a)

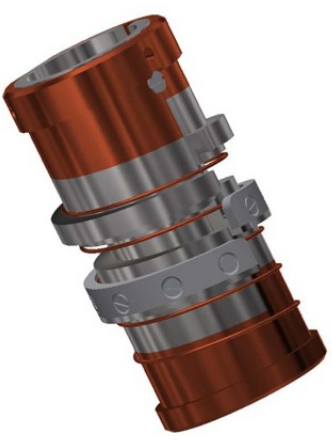

b)

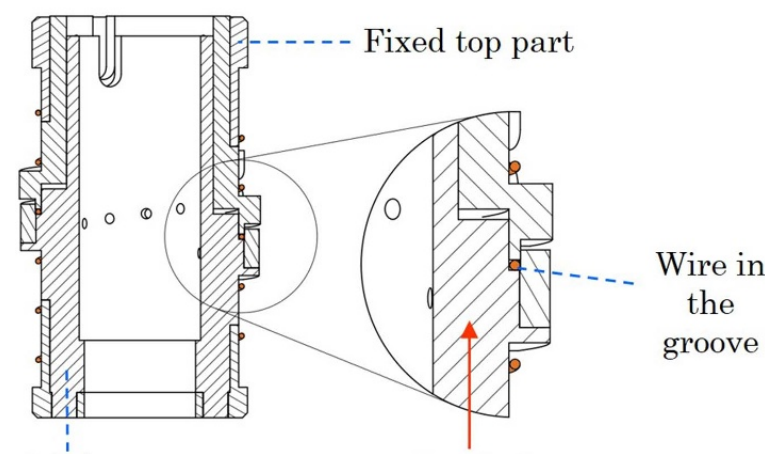

Movable bottom part
Applied

Force

Figure 2. (a) 3D model of the C-WASP device conceived by Seeber et al at University of Geneva. The sample holder is made of Stainless Steel $316 \mathrm{~L}$ and Copper OFHC, (b) cross-sectional cut showing how the force is applied to the superconducting wire.
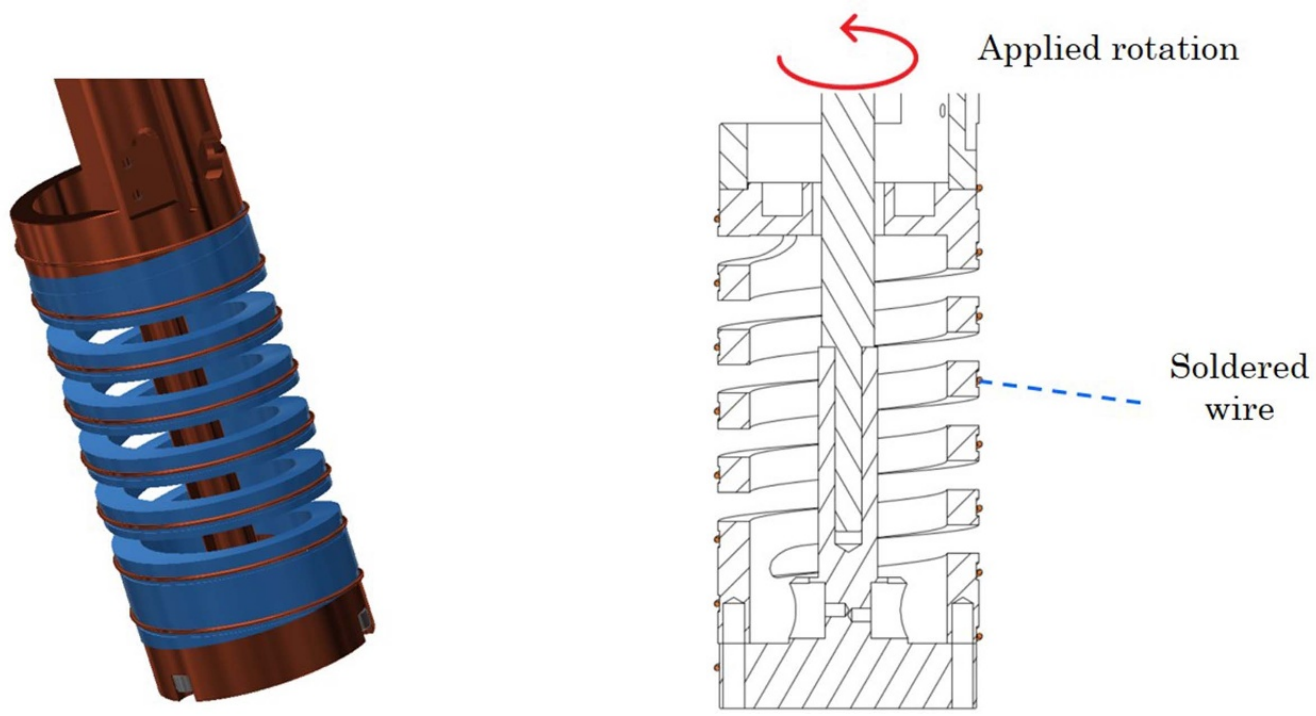

Figure 3. Principle of the Walters spring (WASP) design. The superconducting wire is soldered inside a rectangular-shaped groove. The axial strain is exerted by applying a torque to the spring's upper end. A spring made in $\mathrm{CuBe}$ has been used for the tests reported in this manuscript.

\subsection{Mechanical stress-strain measurements}

At last, the mechanical stress-strain measurements were performed at $4.2 \mathrm{~K}$, using straight wire samples subjected to uni-axial tensile loads. The displacement and the force readings were done using extensometers and load cells respectively.

\section{Results and discussion}

\subsection{The role of axial strain in the response of the wires to transverse loads}

Three round samples were tested under transverse applied loads. Figure 4 reports the results from the critical current measurements performed in the compressive WASP configuration at $4.2 \mathrm{~K}$ and $19 \mathrm{~T}$. The plot displays the evolution of the $I_{c}$ with applied stress using two types of markers: full markers correspond to the value of critical current when the wire is loaded, and empty markers correspond to the value when the load has been removed. The measurement's sequence accounts for the removal of the load each two stress steps, with the objective of analyzing in detail the onset of permanent damage in the wires. The compressive stress value is computed using a purely geometrical relationship,

$$
\sigma=\frac{\text { Force }_{(\text {measured })}}{\text { Width }_{\text {anvil }} \times \text { Length }_{\text {anvil }}},
$$

where the applied force is gradually increased up to a maximum of $35 \mathrm{kN}$, and the area of contact is calculated using width $_{\text {anvil }}=1.15 \mathrm{~mm}$ and length ${ }_{\text {anvil }}=126 \mathrm{~mm}$.

Even though all samples are identical and they were prepared following the same process, the figure shows that they present a different initial critical current $\left(I_{c 0}\right.$ - defined as the critical current at $0 \mathrm{MPa}$ ) and a different electro-mechanical response to transverse stress. The earliest measured sample, called Sample 1, shows an $I_{c 0}=124 \mathrm{~A}$, the second sample 


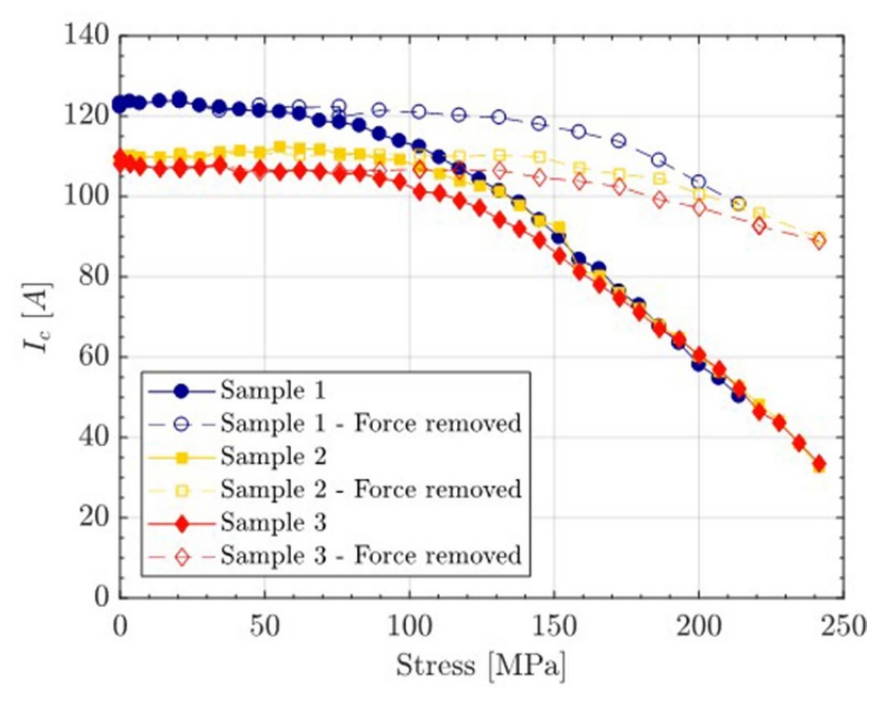

Figure 4. Critical current evolution as function of the applied transverse stress at $19 \mathrm{~T}$. Full markers depict the $I_{c}$ value when the load is being applied, empty markers show the critical current value upon force removal.

Table 2. $B_{c 2}$ values extracted from the C-WASP experiment.

\begin{tabular}{lccc}
\hline & Transverse stress (MPa) & $B_{c 2}^{\text {load }}(\mathrm{T})$ & \multicolumn{2}{c}{$B_{c 2}^{\text {unload }}(\mathrm{T})$} \\
\hline \multirow{3}{*}{ Sample 1 } & 21 & \multicolumn{3}{c}{25.7} \\
& 145 & 24.7 & \multicolumn{2}{c}{25.3} \\
Sample 2 & 214 & 23.3 & \multicolumn{2}{c}{24.85} \\
& 35 & 22.84 & 24.89 \\
Sample 3 & 220 & \multicolumn{3}{c}{24.87} \\
& 35 & 23.55 & 24.5 \\
& 186 & 22 & 24.3 \\
\hline
\end{tabular}

(Sample 2) presents an $I_{c 0}=110 \mathrm{~A}$, and Sample 3 shows the lowest value among the three, with $I_{c 0}=107 \mathrm{~A}$.

With increasing stress levels, all the wires converge to a common behavior under the presence of the transverse load. This phenomenon is clearly identified in figure 4 by the overlap of the three curves represented with solid markers. The implications of such an experimental evidence are twofold: (1) the measurements indicate that there exists an initial difference between the samples and (2) they show that the response of all the wires come to be the same, when the difference is overcome due to the presence of large applied transverse loads $\left(\sigma_{\text {applied }}>160 \mathrm{MPa}\right.$, in this experiment). Interestingly, the same effect is not perceived once the load is removed. Upon force unload, the samples recover part of their original differences. The understanding of this behavior, not seen in previous experimental campaigns [30], constitutes the novelty of this work and sheds new light on the mechanisms behind critical current degradation.

To explain it, let us limit ourselves firstly to the beginning of the measurement, when the wire is not subjected to any transverse mechanical load ( $\sigma=0 \mathrm{MPa}$ ). For a wide range of multifilamentary $\mathrm{Nb}_{3} \mathrm{Sn}$ superconductors, the scaling law for the field dependence of the critical current follows the simplified expression $[33,60]$ :

$$
I_{c}(B, \varepsilon)=C\left(\frac{B}{B_{c 2}(\varepsilon)}\right)^{-0.5}\left(1-\frac{B}{B_{c 2}(\varepsilon)}\right)^{2}
$$

where $C$ is a prefactor shown to be independent of strain over a large range of applied loads [33], $B$ is the magnetic field and $B_{c 2}(\varepsilon)$ is the strain dependent upper critical field. More details about the derivation of the simplified expression in equation 2 from the general scaling law reported in [10] can be found in [60].

In the absence of filament cracks (when there is no reduction of the available superconductor area), and assuming that the lack of applied transverse loads is a sufficient condition to ensure the invariability of $C$, the scaling law suggests that a change in $I_{c}$ for identical wires would imperatively come from a change in their $B_{c 2}$ due to a different mechanical strain state. In the presented case, since no transverse loads are applied, the source of the different strain state must be in the axial component.

We therefore propose that the measured initial deviation in $I_{c}$ between the three wires can be fully explained by a discrepancy in their original axial strain condition, which has its origin in an elastic component introduced during sample mounting. The complete prove for this statement is found in the measurements performed at different magnetic fields for certain stress levels in the C-WASP tests. We could evaluate the upper critical field for the three wires at different applied loads, using the well-known Kramer extrapolation [61]. The results, shown in table 2, support straightforwardly the development presented in the previous paragraph. Sample 1, the one with larger initial critical current, shows an initial $B_{c 2}$ value of 25.7T. On the contrary, Sample 2 and 3 show a value of $24.89 \mathrm{~T}$ and $24.87 \mathrm{~T}$, respectively, in good agreement with their lower measured $I_{c 0}$. The variability in the $B_{c 2}$ determination for different $I_{c}$ measurements has been proven to be lower than $1 \%$.

Besides this conclusive experimental indication, and to further analyze the origin of the $I_{c 0}$ variability between wires, complementary critical current measurements under axial loads were performed in a standard Walters spring configuration. We used a reference wire from the same billet, obtaining the results shown in figure 5(a). We found as well that at 19T the value of $I_{c 0} \varnothing$ strain for the wires is $108 \mathrm{~A}$, very close to the initial $I_{c}$ measured for samples 2 and 3 . Combining both results, the test confirms that in the vicinity of the zero applied strain state (figure 5(b)), $0.05 \%$ axial strain can lead to an increase of $I_{c}$ of the order of 16A. This increment is perfectly in line with the measured difference between Sample 1 and the other two samples, and provides a quantitative explanation for the $I_{c 0}$ variability. To give a visual representation of the test results, the analysis at $19 \mathrm{~T}$ is summarized in figure 5(b). The plot shows a zoom of the WASP measurement, adding all the values of $I_{c 0}$ from the transverse tests as function of their interpolated axial strain level.

In a similar way to the compressive tests, we also studied the field dependence of the critical current in the WASP tests. Critical current measurements at different fields were done for selected axial strain levels. The obtained $B_{c 2}$ values are shown in table 3 . A very similar upper critical field to that of samples 2 and 3 has been found for an axial strain level close to zero 
Table 3. $B_{c 2}$ values extracted from the WASP experiment.

\begin{tabular}{lc}
\hline Wire's axial strain $(\%)$ & $B_{c 2}(\mathrm{~T})$ \\
\hline-0.15 & 22.95 \\
0.03 & 24.7 \\
0.12 & 25.7 \\
0.22 & 26.2 \\
0.31 & 26.1 \\
\hline
\end{tabular}

a)

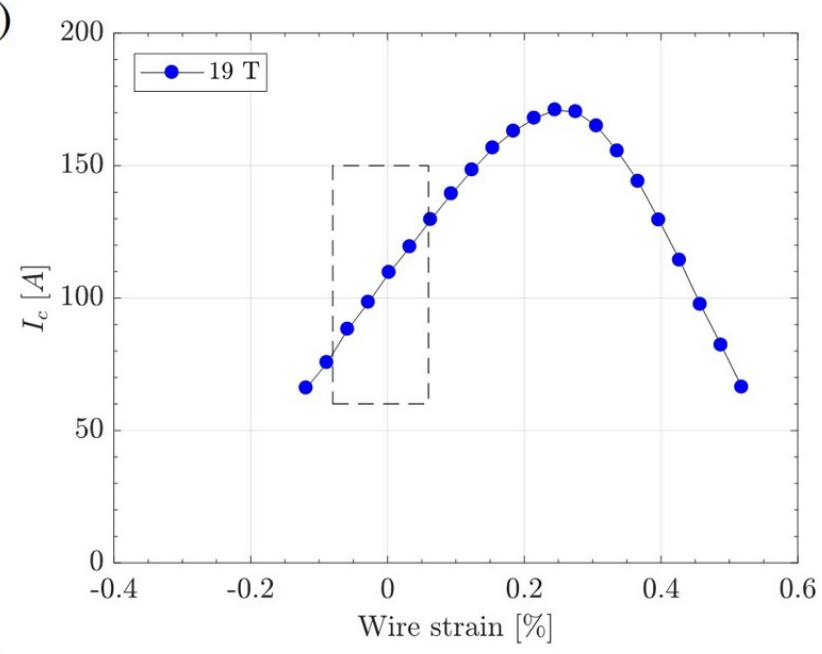

b)

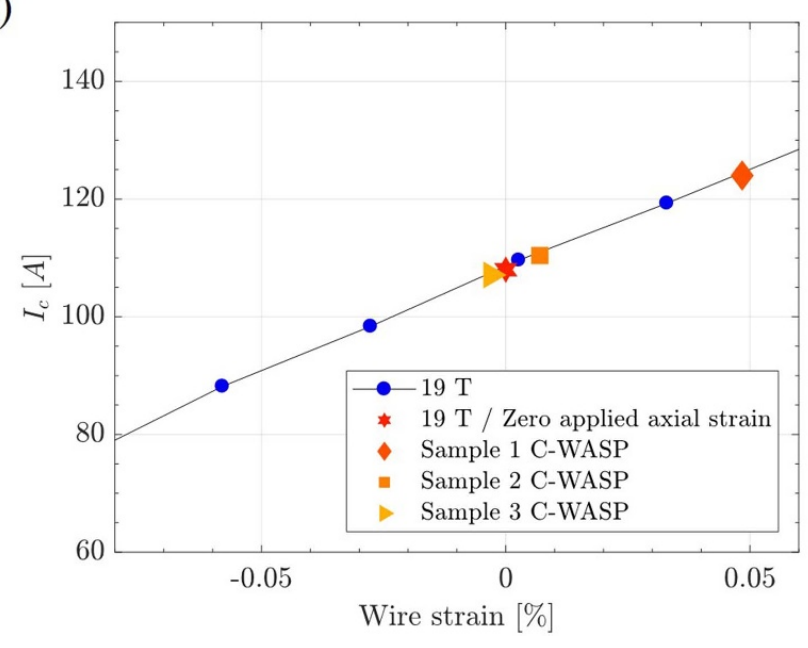

Figure 5. (a) Critical current evolution as function of the applied axial strain for a reference wire measured in the WASP device. Only the measurements at $19 \mathrm{~T}$ are shown for simplicity. The dashed rectangle delimits the zoomed region shown in (b). (b) Detailed zoom of the WASP measurement. Markers display the interpolated values of strain for the three C-WASP samples.

$(0.03 \%)$. On the other hand, a $B_{c 2}$ value close to the one of Sample 1 has been identified at an axial strain level of $0.12 \%$. The results thus confirm the presence of an initial axial tensile component in the strain state of Sample 1, while Samples 2 and 3 are close to a zero level of strain in that direction.

The difference in the axial strain between wires, calculated to be in the range of $0.05 \%-0.09 \%$ in the last two paragraphs, is thought to have its origin in the delicate sample mounting

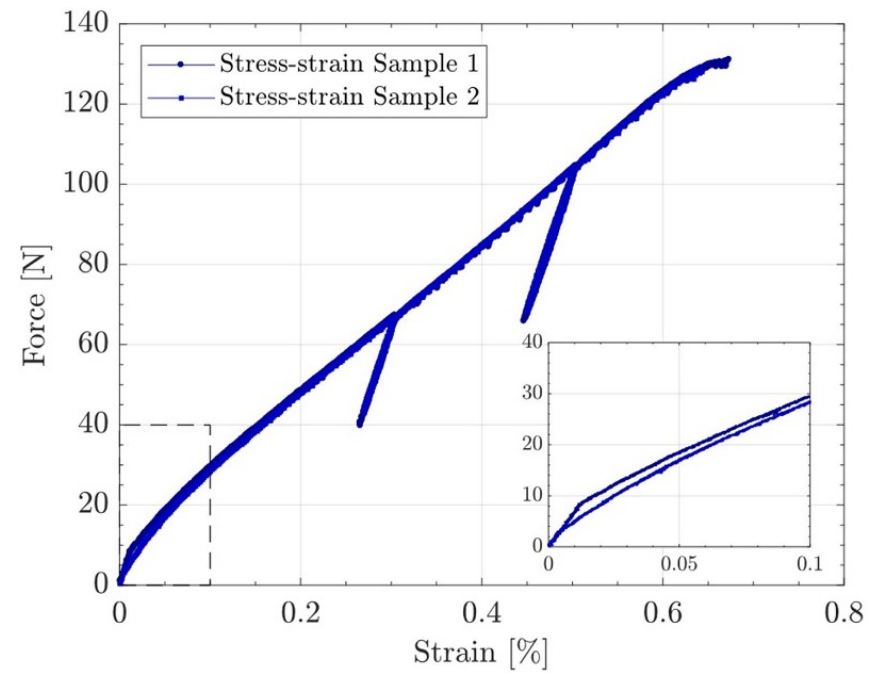

Figure 6. Mechanical stress-strain measurements performed for two straight samples of the investigated wire. The bottom-right box shows a detailed zoom in the area indicated by the dashed rectangle. The force required to induce a strain in the range of $0.05 \%-0.1 \%$ equals $20-30 \mathrm{~N}$.

activities as mentioned before. The wires may be pulled differently during the preparation process, introducing an elastic axial strain component that is kept by the soldering of the wire extremities. Figure 6 shows the result from two stressstrain measurements performed to support the explanation. As it can be seen from the test outcome, an axial force as small as $20-30 \mathrm{~N}$ is enough to reach the required level of strain of $0.05 \%-0.09 \%$.

Our investigation on the novel measured behavior for the three samples is finally completed by reviewing the evolution of the wires' response at increasing stress levels. As already introduced, the elastic axial pre-strain component seems to be released at large applied transverse stress levels when the load is present. This phenomenon could be partially explained by a strain release mechanism resulting from the Poisson effect of the applied transverse load, together with the geometry and particularities of the test setup. Finite Element simulations performed in [62] for wires enclosed in a cavity and subjected to transverse forces, confirm the relevant effects seen in axial direction. A permanent contribution to the release of the axial strain may come as well from the plastic deformation of the stabilizing matrix and from filament cracks. In this regard, several works are available in the community dealing with the plastic release of residual strain for $\mathrm{Nb}_{3} \mathrm{Sn}$ wires [63, 64] Although in our case, the lack of convergence after force removal points out that permanent effects are not the main factor present. The identification of the exact strain release mechanism acting in our test setup is left open for further research and will be tackled in future works.

\subsection{The effect of transverse loads on conductor performance}

Having shown that the axial strain component is behind the observed differences between the wires, the last part of the measurement in figure 4 provides the second piece of essential 
a)

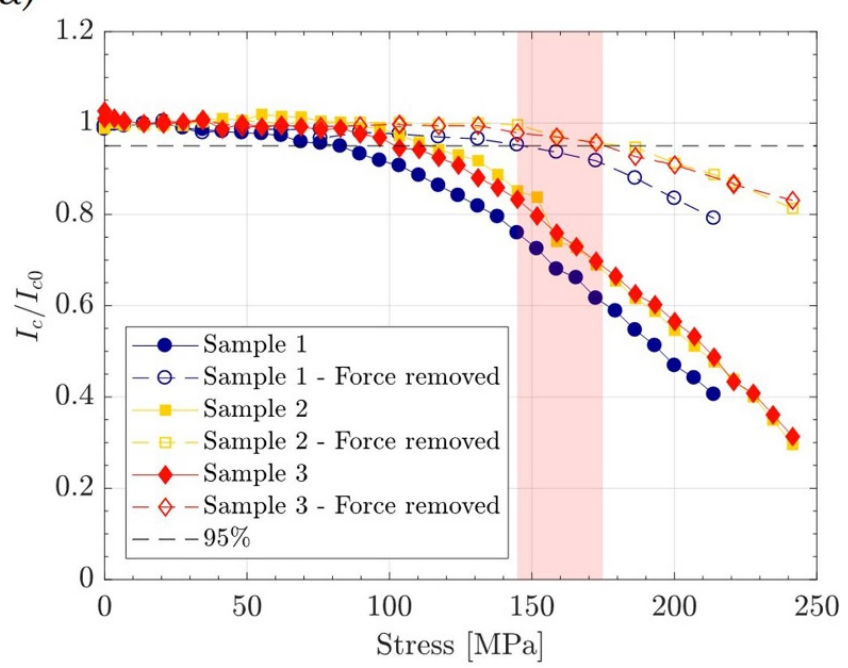

b)

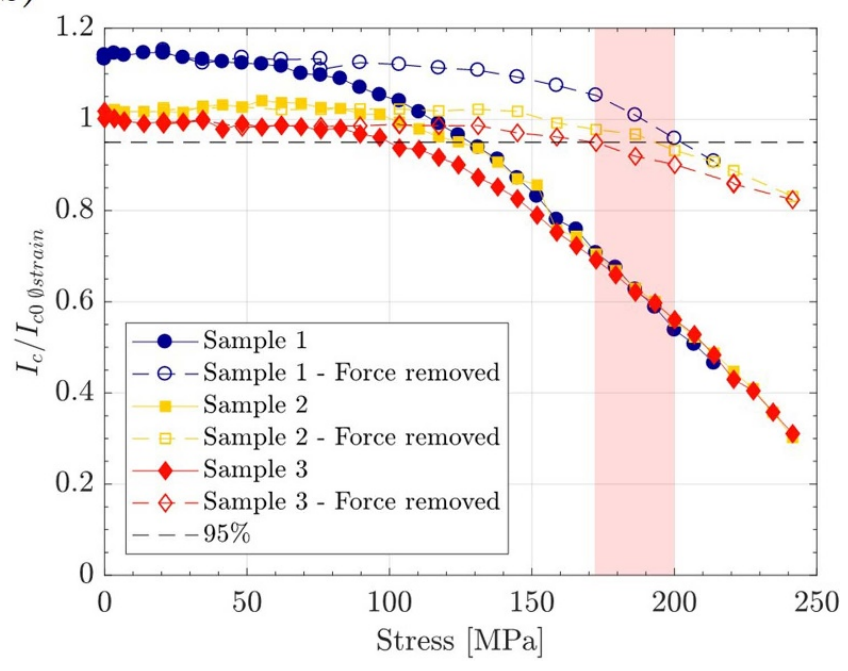

Figure 7. Critical current evolution as function of the applied transverse stress using normalized values. In plot (a), the value for normalization has been chosen to be the $I_{c 0}$ of each wire as explained in the text. Plot (b), instead, shows the results when the common $I_{c 0} \varnothing$ strain for all wires is considered. In both cases, the horizontal dashed line shows the $95 \%$ threshold, while the red box contains the range at which this line intersects the three wires.

information from the study. At high level of stress, $\sigma_{\text {applied }}>$ $160 \mathrm{MPa}$, all samples show the same critical current under load and a certain decrease of $I_{c}$ when the load is removed. From the figure, we can identify the start of the $I_{c}$ decrease after force removal at around 110MPa for sample 1 and at $130 \mathrm{MPa}$ for samples 2 and 3. A permanent reduction in critical current upon force unloading indicates the onset of critical current degradation in the wire. The determination of the experimental value of stress at which this occurs $\left(\sigma_{\text {irr }}\right)$ is an exercise of the highest impact for magnet design. Its value can be conventionally obtained by defining a critical threshold for the fraction of $I_{c}$ recovered after force unloading $\left(I_{c}^{\text {unload }} / I_{c 0}\right)$. Following our standard convention, we propose a threshold value of $5 \%$ at $19 \mathrm{~T}$. Nevertheless, the variation in $I_{c 0}$ for the measured identical wires makes the analysis delicate. Using each

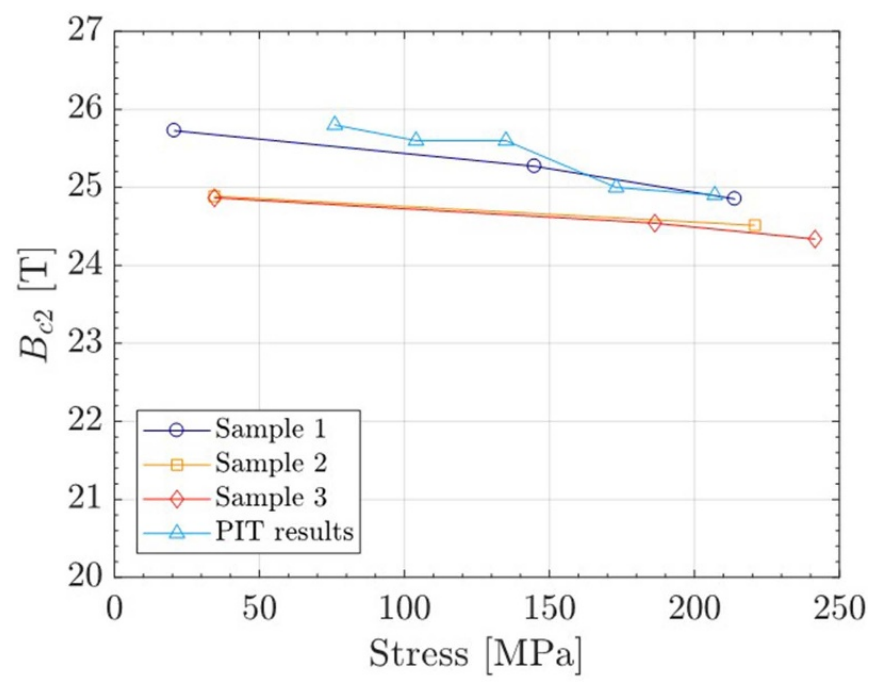

Figure 8. $B_{c 2}$ evolution after force removal for the investigated wires and a PIT sample measured in [30]. The fact that the decrease in $B_{c 2}$ for the RRP wires with zero initial applied strain (Samples 2 and 3 ) is approximately $50 \%$ of the one reported for PIT, may indicate a different degradation mechanism.

wire's initial critical current for normalization, we obtained the following $\sigma_{\text {irr }}$ stress values (figure $7(\mathrm{a})$ ): $\sigma_{\text {irr Sample } 1}=$ $145 \mathrm{MPa}, \sigma_{\text {irr Sample } 2}=175 \mathrm{MPa}$ and $\sigma_{\text {irr Sample } 3}=175 \mathrm{MPa}$. As an alternative, in order to remove from the analysis the influence of axial strain, we propose a second criterion for the computation of a meaningful $\sigma_{\text {irr. }}$. It consists in normalizing the response of the three wires with the value of $I_{c 0}$ at zero applied axial strain, which is common for all samples $\left(I_{c}^{\text {unload }} / I_{c 0} \varnothing\right.$ strain $)$. Using this expedient we can compare the high stress response of the wires, irrespective of the initial state of the sample. In this case, the computed $\sigma_{\text {irr }} \varnothing$ strain lays in the range of $170-200 \mathrm{MPa}$ as seen in figure 7 (b). Note that this is a conventional assumption that we make to compare the results, not necessarily pointing to a physical explanation of the $I_{c}$ reduction.

Lastly, by studying the wires' behavior under load, we found that a transverse stress level of $150 \mathrm{MPa}$ (in line with the stress values expected during operation of new high-field magnet designs) induces an $I_{c}$ reduction at $19 \mathrm{~T}$ of about $16 \%-28 \%$ with respect to each wire's initial critical current $\left(I_{c}^{\mathrm{load}} / I_{c 0}\right)$. Though the reduction at lower fields would be smaller, the plot highlights the essential role of mechanics in the performance of these wires, and motivates its impact in magnet design. A complete summary of the results is shown in table 4 .

\subsection{An insight into the degradation mechanisms of RRP $\mathrm{Nb}_{3} \mathrm{Sn}$ wires}

To conclude the analysis, we investigated the full evolution of the upper critical field along the transverse loading history. This study adds very valuable information on the mechanisms causing the critical current degradation. The most relevant data can be extracted from the permanent effects in $B_{c 2}$ remaining after load removal. As already mentioned in the introduction, the irreversible decrease of the critical current under transverse 
Table 4. Summary on wires' results.

\begin{tabular}{llllll}
\hline Sample & $I_{c 0}(19 \mathrm{~T})$ & $I_{c 0}(16 \mathrm{~T})$ & Initial $B_{c 2}$ & $\sigma_{\text {irr }}$ & $\sigma_{\text {irr } \varnothing \text { strain }}$ \\
\hline Sample 1 (S1) & $124 \mathrm{~A}$ & $283.1 \mathrm{~A}$ & $25.7 \mathrm{~T}$ & $145 \mathrm{MPa}$ & $200 \mathrm{MPa}$ \\
Sample 2 (S2) & $110.4 \mathrm{~A}$ & $274.5 \mathrm{~A}$ & $24.89 \mathrm{~T}$ & $175 \mathrm{MPa}$ & $190 \mathrm{MPa}$ \\
Sample 3 (S3) & $107.1 \mathrm{~A}$ & $268.3 \mathrm{~A}$ & $24.87 \mathrm{~T}$ & $175 \mathrm{MPa}$ & $172.5 \mathrm{MPa}$ \\
Sample axial & $108 \mathrm{~A}$ & & & \\
\hline
\end{tabular}

loads results from the combination of two effects: the plastic deformation of the $\mathrm{Cu}$ matrix, and the breakage of the $\mathrm{Nb}_{3} \mathrm{Sn}$ filaments. The effects of these two phenomena on the critical current are different. On one side, the effect of residual strain decreases the wire's $B_{c 2}$ due to a deformation of the $\mathrm{Nb}_{3} \mathrm{Sn}$ crystal structure. On the other side, filament cracks just reduce the available area of superconductor leaving $B_{c 2}$ almost unaltered. Figure 8 summarizes the results obtained for the $B_{c 2}$ evolution of the investigated samples, and adds for comparison the example of a PIT wire published in [30]. The behavior of Sample 1 is understood in terms of its initial tensile axial strain component and the mechanical strain release mechanism described before. The cyclic loading seems to release the initial strain, slowly converging to the $B_{c 2}$ values of the two other samples. Therefore, for the sake of a fair comparison, only the measurements of the wires with an initial zero applied axial strain state, which include the PIT sample, are considered in the analysis. Under the same range of pressure, the decrease in $B_{c 2}$ is considerably smaller for the two RRP samples with zero initial applied strain (Samples 2 and 3) than for their PIT counterpart, approximately $50 \%$. The reduced impact in $B_{c 2}$ upon force unloading for these specific samples may point to a small effect of residual plastic strain in the copper matrix. Unlike in the PIT wire, the permanent reduction of $I_{c}$ for the two RRP samples seems to be driven by the breakage of superconducting filaments. We plan to investigate further this result, which could have important implications for magnet design.

\section{Conclusions}

This manuscript presents the results from an experimental campaign aimed at characterizing the electro-mechanical response of state-of-the-art $\mathrm{RRP} \mathrm{Nb}_{3} \mathrm{Sn}$ wires to mechanical loads. With the objective of obtaining relevant information on magnet performance from a single wire experiment, we investigated the effect of loads applied independently in transverse and axial directions. Special emphasis was put in the understanding of the effect of the initial axial strain in the wire's response to transverse loads. The main outcome from the work is enumerated next:

(a) We identified a remarkable effect of the initial axial strain state in the wire's response to transverse loads. The axial component determines the initial critical current and its evolution at low transverse stress levels.

(b) At high stress levels, and while the force is being applied $\left(\sigma_{\text {applied }}>160 \mathrm{MPa}\right.$ in this experiment $)$, the effect of the axial strain component is overcome and the transverse load becomes dominant.
In this regime all wires show the same critical current.

(c) Critical current tests under axial loads served to provide the needed complimentary information for the study. The results shown in this manuscript suggest that it is always essential to consider the complete strain tensor in the analysis of the electro-mechanical limits of $\mathrm{Nb}_{3} \mathrm{Sn}$ superconductors.

(d) The transverse stress value leading to a conventional permanent reduction in $I_{c}$ of $5 \%$, has been found to be in the range of 145-175 $\mathrm{MPa}$. The different initial axial strain state is behind the range obtained for the three samples of the same wire.

(e) By studying the wires' response at different magnetic fields, we found a very limited permanent decrease in the upper critical field $\left(B_{c 2}\right)$ resulting from transverse stresses up to $250 \mathrm{MPa}$. This fact suggests that the dominating mechanism behind critical current degradation, for the investigated wires, is the breakage of superconducting filaments.

\section{Acknowledgements}

UNIGE acknowledges financial support from the European Organization for Nuclear Research (CERN), Memorandum of Understanding for the FCC Study Addendum FCC-GOV-CC0176 (KE 4612/ATS). This work was supported in part by the European Union's Horizon 2020 research and innovation programme under Grant 654305, EuroCirCol project.

\section{ORCID iDs}

J Ferradas Troitino (D) https://orcid.org/0000-0001-78749722

T Bagni (D) https://orcid.org/0000-0001-8654-783X

C Senatore (D) https://orcid.org/0000-0002-9191-5016

\section{References}

[1] Ballarino A and Bottura L 2015 Targets for R\&D on $\mathrm{Nb}_{3} \mathrm{Sn}$ conductor for high energy physics IEEE Trans. Appl. Supercond. 256000906

[2] Schoerling D and Zlobin A $2019 \mathrm{Nb}_{3}$ Sn Accelerator Magnets Particle Acceleration and Detection (Berlin: Springer, Cham) (https://doi.org/10.1007/978-3-030-16118-7)

[3] Rossi L and Bruning O 2015 The High Luminosity Large Hadron Collider Advanced Series on Directions in High 
Energy Physics Vol 24 (Singapore: World Scientific) (https://doi.org/10.1142/9581)

[4] FCC-hh: The Hadron Collider 2019 Eur. Phys. J. Spec. Top. 228 755-1107

[5] Cooley L D 2018 More time for Nb3Sn magnet conductors 100501

[6] Cooley L D, Ghosh A K, Dietderich D R and Pong I 2017 Conductor specification and validation for high-luminosity LHC quadrupole magnets IEEE Trans. Appl. Supercond. 276000505

[7] Parrell J A, Zhang Y, Field M B, Meinesz M, Huang Y, Miao H, Hong S, Cheggour N and Goodrich L 2009 Internal tin $\mathrm{Nb}_{3}$ Sn conductors engineered for fusion and particle accelerator applications IEEE Trans. Appl. Supercond. 192573

[8] Field M B, Zhang Y, Miao H, Gerace M and Parrell J A 2014 Optimizing $\mathrm{Nb}_{3}$ Sn conductors for high field applications IEEE Trans. Appl. Supercond. 246001105

[9] Sanabria C, Field M, Lee P J, Miao H, Parrell J and Larbalestier D C 2018 Controlling Cu-Sn mixing so as to enable higher critical current densities in RRP $\mathrm{Nb}_{3} \mathrm{Sn}$ wires Supercond. Sci. Technol. 31064001

[10] Ekin J W 2010 Unified scaling law for flux pinning in practical superconductors: I. Separability postulate, raw scaling data and parametrization at moderate strains Supercond. Sci. Technol. 23083001

[11] Ekin J W 1980 Strain scaling law for flux pinning in practical superconductors. Part 1: Basic relationship and application to $\mathrm{Nb}_{3} \mathrm{Sn}$ conductors Cryogenics 20 611-24

[12] Ekin J W 2006 Experimental Techniques for Low-Temperature Measurements: Cryostat Design, Material Properties and Superconductor Critical current Testing (New York: Oxford University Press) (https://doi.org/10.1093/acprof:oso/9780 198570547.001.0001)

[13] Oh S and Kim K 2006 A scaling law for the critical current of $\mathrm{Nb}_{3} \mathrm{Sn}$ strands based on strong-coupling theory of superconductivity J. Appl. Phys. 99033909

[14] Summers L T et al 1991 A model for the prediction of $\mathrm{Nb}_{3} \mathrm{Sn}$ critical current as function of field, temperature, strain and radiation damage IEEE Trans. Magn. 27 2041-44

[15] Lu X F, Taylor D M and Hampshire D P 2008 Critical current scaling laws for advanced $\mathrm{Nb}_{3} \mathrm{Sn}$ superconducting strands for fusion applications with six free parameters Supercond. Sci. Technol. 21105027

[16] Arbelaez D, Godeke A and Prestemon S O 2009 An improved model for the strain dependence of the superconducting properties of $\mathrm{Nb}_{3} \mathrm{Sn}$ Supercond. Sci. Technol. 22025005

[17] Taylor D M J and Hampshire D P 2005 The scaling law for the strain dependence of the critical current density in $\mathrm{Nb}_{3} \mathrm{Sn}$ superconducting wires Supercond. Sci. Technol. 18 S241

[18] ten Haken B, Godeke A and ten Kate H H 1995 The influence of compressive and tensile axial strain on the critical properties of $\mathrm{Nb}_{3} \mathrm{Sn}$ conductors IEEE Trans. Appl. Supercond. 5 1909-12

[19] ten Haken B, Godeke A and ten Kate H H J 1999 The strain dependence of the critical properties of $\mathrm{Nb}_{3} \mathrm{Sn}$ conductors $J$. Appl. Phys. 85 3247-53

[20] Godeke A, ten Haken B and ten Kate H H 2002 The deviatoric strain description of the critical properties of $\mathrm{Nb}_{3} \mathrm{Sn}$ conductors Physica C 372-376 1295-8

[21] Godeke A, ten Haken B, ten Kate H H and Larbalestier D C 2006 A general scaling relation for the critical current density in $\mathrm{Nb}_{3} \mathrm{Sn}$ Supercond. Sci. Technol. 19 R100

[22] Markiewicz W D 2004 Elastic stiffness model for the critical temperature $\mathrm{Tc}$ of $\mathrm{Nb}_{3} \mathrm{Sn}$ including strain dependence Cryogenics 44 767-82

[23] Markiewicz W D 2006 Invariant temperature and field strain functions for $\mathrm{Nb}_{3} \mathrm{Sn}$ composite superconductors Cryogenics $46846-63$
[24] Markiewicz W D 2008 Comparison of strain scaling functions for the strain dependence of composite $\mathrm{Nb}_{3} \mathrm{Sn}$ superconductors Supercond. Sci. Technol. 21054004

[25] Godeke A, Hellman F, Ten Kate H H and Mentink M G T 2018 Fundamental origin of the large impact of strain on superconducting $\mathrm{Nb}_{3}$ Sn Supercond. Sci. Technol. 31105011

[26] Bordini B, Alknes P, Bottura L, Rossi L and Valentinis D 2013 An exponential scaling law for the strain dependence of the $\mathrm{Nb}_{3} \mathrm{Sn}$ critical current density Supercond. Sci. Technol. 26075014

[27] Bottura L et al $2009 J_{c}(B, T, \varepsilon)$ Parameterization for the ITER $\mathrm{Nb}_{3} \mathrm{Sn}$ production IEEE Trans. Appl. Supercond. 19 $1521-4$

[28] Calzolaio C et al 2015 Electro-mechanical properties of PIT $\mathrm{Nb}_{3} \mathrm{Sn}$ wires under transverse stress: experimental results and FEM analysis Supercond. Sci. Technol. 28055014

[29] Tommasini D et al 2017 The 16 T dipole development program for FCC IEEE Trans. Appl. Supercond. 274000405

[30] Gamperle L et al 2020 Determination of the electromechanical limits under transverse stress of high-performance $\mathrm{Nb}_{3} \mathrm{Sn}$ Rutherford cables from a single-wire experiment Phys. Rev. Res. 2013211

[31] Godeke A 2018 The history of Transverse Pressure Tests of $\mathrm{Nb}_{3}$ Sn Rutherford Cables, in Workshop on $\mathrm{Nb}_{3} \mathrm{Sn}$ technology for accelerator magnets, Paris, France (available at: https://indico.cern.ch/event/743626/contributions/ $3154559 /)$

[32] Ekin J W 1987 Effect of transverse compressive stress on the critical current and upper critical field of $\mathrm{Nb}_{3} \mathrm{Sn} J$. Appl. Phys. 62 4829-34

[33] Bordini B, Alknes P, Ballarino A, Bottura L and Oberli L 2014 Critical current measurements of high- $\mathrm{Jc} \mathrm{Nb}_{3} \mathrm{Sn}$ Rutherford cables under transverse compression IEEE Trans. Appl. Supercond. 249501005

[34] Duvauchelle J E, Bordini B, Fleiter J and Ballarino A 2018 Critical current measurements under transverse pressure of a $\mathrm{Nb}_{3} \mathrm{Sn}$ Rutherford cable based on $1 \mathrm{~mm}$ RRP wires IEEE Trans. Appl. Supercond. 284802305

[35] Dietderich D R, Scanlan R M, Walsh R P and Miller J R 1999 Critical current of superconducting Rutherford cable in high magnetic fields with transverse pressure IEEE Trans. Appl. Supercond. 9 122-5

[36] Barzi E 2002 A device to test critical current sensitivity of $\mathrm{Nb}_{3}$ Sn cables to pressure Proc. AIP Conf. Proc. 614 45-52

[37] Barzi E, Turrioni D and Zlobin A V 2008 Effect of transverse pressure on brittle superconductors IEEE Trans. Appl. Supercond. 18 980-3

[38] Seeber B, Ferreira A, Abächerli V, Boutboul T, Oberli L and Flükiger R 2007 Transport properties up to $1000 \mathrm{~A}$ of $\mathrm{Nb}_{3} \mathrm{Sn}$ wires under transverse compressive stress IEEE Trans. Appl. Supercond. 17 2643-6

[39] ten Haken B, Zaitseva T N and ten Kate H H J 1994 Modeling of strain in multifilamentary wires deformed by thermal contraction and transverse forces Cryogenics 34 513-16

[40] Jakob B and Pasztor G 1989 Effect of transverse compressive stress on the critical current of cabled $\mathrm{Nb}_{3} \mathrm{Sn}$ conductor IEEE Trans. Magn. 25 2379-81

[41] Boschman H, Verweij A P, Wessel S, ten Kate H H J and van de Klundert L J M 1991 The effect of transverse loads up to $300 \mathrm{MPa}$ on the critical currents of $\mathrm{Nb}_{3}$ Sn cables IEEE Trans. Magn. 27 1831-4

[42] ten Kate H H, Weijers H W and van Oort J M 1993 Critical current degradation in $\mathrm{Nb}_{3} \mathrm{Sn}$ cables under transverse pressure IEEE Trans. Appl. Supercond. 3 1334-7

[43] Gao P et al 2018 Transverse pressure dependence of critical current in RRP and PIT type $\mathrm{Nb}_{3}$ Sn Rutherford cables for use in future accelerator magnets Proc. Appl. Supercond. Conf. seattle Presentation no.: 3LPo1D-09 
[44] Gao P 2019 PhD Thesis University of Twente Transverse pressure effect on superconducting $\mathrm{Nb}_{3} \mathrm{Sn}$ Rutherford and ReBCO Roebel cables for accelerator magnets (https://doi.org/10.3990/1.9789402816587)

[45] ten Kate H H J, Weijers H W and van Oort J M 1993 Critical current degradation in $\mathrm{Nb}_{3} \mathrm{Sn}$ cables under transverse pressure IEEE Trans. Appl. Supercond. 31334

[46] Barzi E, Wokas T and Zlobin A V 2005 Sensitivity of $\mathrm{Nb}_{3} \mathrm{Sn}$ Rutherford-type cables to transverse pressure IEEE Trans. Appl. Supercond. 151541

[47] Cheggour N et al 2019 Implications of the strain irreversibility cliff on the fabrication of particle-accelerator magnets made of restacked-rod-process $\mathrm{Nb}_{3} \mathrm{Sn}$ wires $\mathrm{Sci}$ Rep 95466

[48] Cheggour N et al 2018 Precipitous change of the irreversible strain limit with heat-treatment temperature in $\mathrm{Nb}_{3} \mathrm{Sn}$ wires made by the restacked-rod process Sci Rep 813048

[49] Ebermann P et al 2019 Influence of transverse stress exerted at room temperature on the superconducting properties of $\mathrm{Nb}_{3}$ Sn wires Supercond. Sci. Technol. 32095010

[50] Ebermann $\mathrm{P}$ et al 2018 Irreversible degradation of $\mathrm{Nb}_{3} \mathrm{Sn}$ Rutherford cables due to transverse compressive stress at room temperature Supercond. Sci. Technol. 31065009

[51] Senatore C 2020 Determination of the electromechanical limits of high-performance $\mathrm{Nb}_{3} \mathrm{Sn}$ Rutherford cables under transverse stress from a single-wire experiment (available at: https://indico.cern.ch/event/896755/attachments/ 2028702/3397208/Senatore_SeminarCERN_Indico.pdf)

[52] Ferracin P et al 2014 Magnet design of the $150 \mathrm{~mm}$ aperture low- $\beta$ quadrupoles for the high luminosity LHC IEEE Trans. Appl. Supercond. 244002306

[53] Ferracin P et al 2015 Development of MQXF: the $\mathrm{Nb}_{3} \mathrm{Sn}$ low- $\beta$ quadrupole for the HiLumi LHC IEEE Trans. Appl. Supercond. 264000207

[54] Seeber B et al 2007 Transport properties up to 1000 A of $\mathrm{Nb}_{3} \mathrm{Sn}$ wires under transverse compressive stress IEEE Trans. Appl. Supercond. 17 2643-6
[55] Mondonico G et al 2012 Effect of quasi-hydrostatical radial pressure on Ic of $\mathrm{Nb}_{3} \mathrm{Sn}$ wires Supercond. Sci. Technol. 25115002

[56] Meyer M 2018 Mechanical characterization of CTD 101K and Stycast 2850 FT epoxy resin systems, CERN. EDMS $1890057 \mathrm{v} 1$ (available at: https://edms.cern.ch/ ui/\#!master/navigator/document?D:100044996: 100044996:subDocs)

[57] Crouvizier M 2018 Impregnated epoxy flexural tests, CERN. EDMS 1902476 v1 (available at: https://edms.cern.ch/ ui/\#!master/navigator/document?D:100059980:100059980: subDocs)

[58] Seeber B et al 2005 Critical current versus strain measurement up to $21 \mathrm{~T}$ and $1000 \mathrm{~A}$ of long length superconducting wires and tapes Rev. Sci. Instrum. $\mathbf{7 6} 093901$

[59] Mondonico G 2013 PhD Thesis Univ. Genève no. Sc. 4529 Analysis of electromechanical properties of A15 type superconducting wires submitted to high mechanical loads (https://doi.org/10.13097/archive-ouverte/unige:26700)

[60] Bordini B 2019 Reversible $\mathrm{I}_{\mathrm{c}}$ reduction of $\mathrm{Nb}_{3}$ Sn Rutherford cables subjected to transverse pressure: impact on the performance of the HL-LHC 11 T dipole (available at: https://indico.cern.ch/event/796352/contributions/3309027/ attachments/1791726/2919254/2019_February_ 6_-_Transverse.pdf)

[61] Kramer E J 1973 Scaling laws for flux pinning in hard superconductors J. Appl. Phys. 441360

[62] Cattabiani A et al 2020 A 3D finite element model of the reversible critical current reduction due to transverse load in $\mathrm{Nb}_{3} \mathrm{Sn}$ Wires IEEE Trans. Appl. Supercond. 308401006

[63] Awaji S, Watanabe K and Katagiri K 2003 Improvement of mechanical and superconducting properties in $\mathrm{CuNb} /(\mathrm{Nb}, \mathrm{Ti})_{3} \mathrm{Sn}$ wires by applying bending strain at room temperature Supercond. Sci. Technol. 16 733-8

[64] Oguro H, Awaji S, Watanabe K, Sugimoto M and Tsubouchi H 2014 Prebending effect for mechanical and superconducting properties of $\mathrm{Nb}$-rod-processed $\mathrm{Cu}-\mathrm{Nb}$ internal-reinforced $\mathrm{Nb}_{3}$ Sn wires IEEE Trans. Appl. Supercond. 248401004 